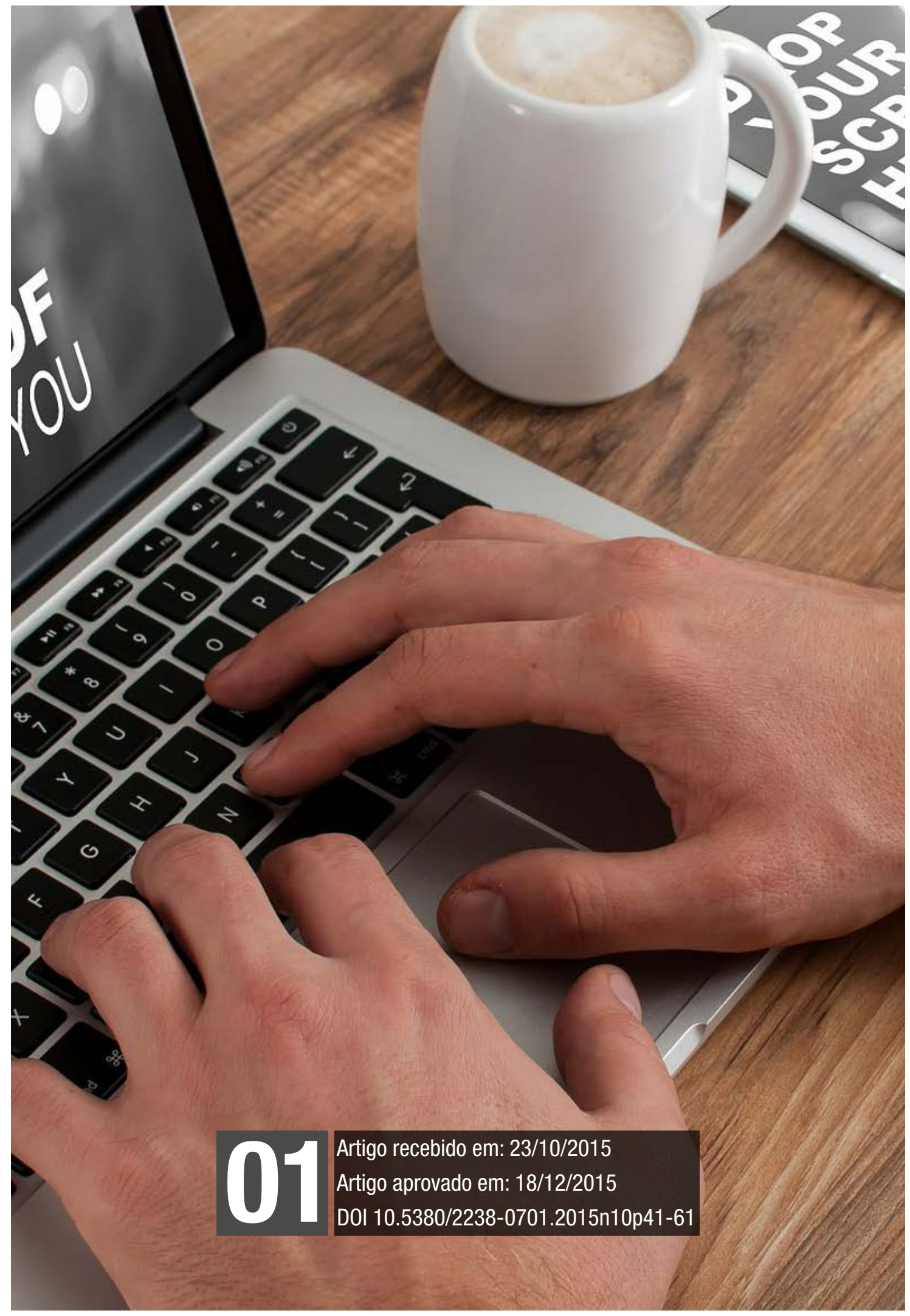


AÇÃO MIDIÁTICA, n. 10. Ju/Dez. 2015. Curitiba. PPGCOM-UFPR. ISSN 2238-0701 


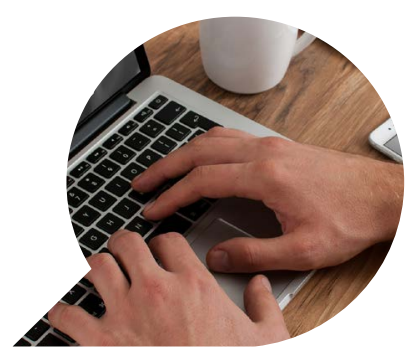

\title{
Nouveau journalisme, nouveau public? Le cas du Huffington Post
}

\author{
Novo jornalismo, novo público? O caso do Huffington Post
}

New journalism, new public? The Huffington Post case

\section{JEAN-CLAUDE SOULAGES}

Résumé: L'irruption du digital dans le domaine de l'information en moins de deux décennies a profondément bouleversé l'univers des médias. Dans le domaine du journalisme, ce changement s'est accompagné de phénomènes de ruptures mais aussi de phénomènes de continuité et de convergences. A côté de ruptures générationnelles, matérielles, économiques d'autres sont plus marquantes, comme la cohabitation dans les médias en ligne comme le Huffington Post de journalistes et de profanes. Se sont imposées des processus de convergence transmédia et crossmédia et simultanément une ubiquité et une accessibilité inédites à l'information de la part de nouveaux publics. Cette reconfiguration de la communication publique et privée liée à l'hégémonie croissante d'Internet nous pousse à une remise en cause inéluctable tout autant de la notion de média que de celle de public. Le journal en ligne

\footnotetext{
* Professeur à l'Université Lumière Lyon 2, et chercheur au Centre Max Weber UMR 5283, il est spécialiste de l'analyse des discours médiatiques et des productions culturelles. jean-claude. soulages@univ-lyon2.fr
} 
s'est transformé en un texte-parcours qui repose sur une nouvelle pratique de consommation, d'interrogation et de vérification de l'information, pratique indexée et tracée à laquelle le public, consciemment ou à son insu, prend sa part œuvrant ainsi à la visibilité et la hiérarchie de l'événement.

Mots-clés: Média; Information; Internet; Digital; Huffington Post.

Resumo: A chegada da digitalização no campo da informação alterou profundamente o universo das mídias, em menos de duas décadas. Na área do jornalismo, esta mudança foi acompanhada de fenômenos de ruptura, mas também de fenômenos de continuidade e de convergências. Ao lado de rupturas generacionais, materiais, econômicas, outras são mais marcantes para o campo, como a convivência nas mídias online, como o The Huffington Post, de jornalistas e amadores. Processos de convergência transmídia e crossmídia convivem simultaneamente com uma ubiquidade e uma acessibilidade inédita da informação por parte dos novos públicos. Esta reconfiguração da comunicação pública e privada conectada com a hegemonia crescente da Internet nos força a um inevitável questionamento tanto sobre a noção de mídia quando sobre a de público. O jornal online se transformou em um texto-percurso que repousa sobre uma nova prática de consumo, interrogação e checagem da informação, prática indexada e para a qual o público, de forma consciente ou não, toma sua parte, trabalhando na visibilidade e na hierarquia do acontecimento.

Palavras-chave: Mídia; Notícias; Internet; Digital; Huffington Post.

Abstract: The arrival of the digital in the field of the information within two decades profoundly upset the universe of the media. In the field of journalism, this change came along with phenomena of disruptions but also with phenomena of continuity and convergences. Next to generational, material, economic breaks, others changes are more striking, as the cohabitation in the me- 
dia on-line as the Huffington Post of journalists and laymen. Processes of convergence transmedia and crossmedia appeared and simultaneously a new ubiquity and an accessibility to the information for of new public. This reconfiguration of the public and private communication connected to the increasing hegemony of Internet pushes us to an inevitable questioning just as much of the notion of media than that of public. The on-line newspaper was transformed into a text-route which bases on a new practice of consumption, interrogation and check of the information, indexed and to which the public, consciously or without knowing, takes his part, working in the visibility and the hierarchy of the event.

Keywords: Media; News; Internet; Digital; Huffington Post. 


\section{Introduction}

L'irruption du digital dans le domaine de la communication en moins de deux décennies a profondément bouleversél'univers des industries culturelles comme celui des médias d'information. Les mutations qui s'en sont suivi sont non seulement révélatrices de bouleversements technologiques et économiques de grande ampleur mais aussi de transformations dans les usages des publics et indirectement dans les routines des professionnels qui sont contraints de revisiter pour la plupart leurs modèles éditoriaux. Si cette hégémonie de la communication digitale est massive, c'est surtout parce que l'appropriation des technologies numériques par les publics a été très rapide. Beaucoup plus rapide que pour la télévision qui a mis 30 ans environ pour s'installer dans le mediascape des français, alors que seulement 15 ans ont suffi pour Internet. Cette appropriation s'est caractérisée et se caractérise toujours par un autre écart par rapport à l'apparition des technologies antérieures. Premier constat dans le domaine du journalisme, même pour cette bien mal nommée "révolution digitale", ce changement s'accompagne de phénomènes de ruptures ces disruptions mises en exergue par la littérature anglo-saxonnemais aussi de phénomènes de continuité et de convergences. Bien sûr, pour beaucoup de chercheurs, les ruptures sont évidentes, par contre les phénomènes de continuité ou de convergence sont le plus souvent sous-estimés.

\section{Les ruptures}

La première rupture a trait à un nouveau rapport à la connaissance et à la culture qui s'accompagne fréquemment d'une quasi-rupture générationnelle et d'un certain nombre d'appréhensions. Sur ce point, le monde digital correspondrait à l'entrée dans une « culture préfigurative » que l'anthropologue Margaret Mead nous annonçait, et au cœur de laquelle la transmission culturelle et les nouveaux usages passent d'abord par les jeunes générations, aujourd'hui les digital natives, pour se transmettre par la suite aux plus anciennes... si toutefois elles se transmettent (Mead, 1971). A tel point que nous sommes constamment dans l'attente d'une sorte d'héritage de l'avenir concernant notre culture, nos savoirs et nos pratiques futures. C'est bien du reste sans cesse cette référence à l'avenir qui nous pousse, depuis leur appari- 
tion, à apprivoiser ces technologies: bientôt pour demain les objets connectés, les big data, la voiture sans conducteur, l'univers des robots, le théorème de Moore $^{1}$, demain, demain, etc. Et les imprécateurs de ce regard saisi par notre futur deviennent les prescripteurs de l'entrée dans cette nouvelle culture et donc indirectement les instigateurs de ruptures avec nos anciens univers de référence et les responsables de cet « avenir de l'oubli » que Raffaele Simone redoute qui nous conduit à reléguer au magasin des accessoires l'héritage des générations passées ; le disque, le livre, la télévision, et peut être demain nos médias d'information et certaines de nos valeurs, etc (Simone, 2012).

Autre rupture dans cet univers de la communication digitale, la cohabitation désormais possible et parfois, comme dans le cas du Huffington Post, sur les mêmes supports entre discours de professionnels de l'information et paroles de profanes. Cohabitation relative et bien sûr négociée dans le monde du journalisme mais effective et massive sur la toile, couronnant pour Patrice Flichy ce " sacre de l'amateur " puisque l'usager peut, à son tour, interagir à tout moment et devenir un acteur dans la production des contenus (Flichy, 2010). Les audiences, public et partenaire lointain que la communication de masse avait laborieusement façonnées et apprivoisées, délaissent les coulisses du média pour s'imposer sur la scène des pages-écrans et faire irruption dans l'espace public.

Seconde rupture mais cette fois-ci d’ordre quasi ontologique. La digitalisation et la dématérialisation des contenus entraînent du même coup leur démultiplication à l'infini, accompagnée de la disponibilité et de la circulation globales des productions digitales et des informations désormais accessibles à tous et partout. Plus besoin de passer par le kiosque pour lire son journal ou par le journal télévisé pour accéder à l'actualité. Les médias sont désormais déliés des supports physiques et des circuits de distribution et leurs usagers devenus un public diasporique actif doué d'un authentique feed back avec toutefois un codicille fâcheux concernant l'attitude de cet usager, le coût invisible, à ses yeux, de l'information et une mauvaise habitude, celle de la quasi gratuité des contenus du web. Le nouvel environnement repose, comme le constate Jérémy Rifkin, sur une «culture de l'accès » qui, mine et démonétise durablement la propriété indi-

1 La loi de Gordon E. Moore établit une croissance exponentielle de la puissance des microprocesseurs (qui doublait tous les 6 mois dans les années 80, aujourd'hui tous les deux ans) 
viduelle jusque là assurée par une valeur d'échange contractuelle et sanctionnée par l'économie de marché (Rifkin, 2005).

Cette même économie de marché qui connaît un autre type de rupture, celle qu'introduit la logique schumpetérienne des cycles économiques : cette logique de destruction créative telle que Karl Marx l'avait observée le premier, basée sur la nécessité pour le capitalisme de révolutionner sans cesse ses moyens de production. Dans cette nouvelle économie, ce sont désormais les datas - et non plus les matières premières qu'il s'agissait à l'époque de transformer en marchandises et en valeur-, ces datas innombrables auxquels aujourd'hui il s'agit de conférer une valeur d'échange monnayable. Sur ce point, la toile constitue sans aucun doute un territoire et une opportunité formidable, puisque ce réseau des réseaux tisse un lien (quasi spontané et gratuit) de tous à tous qui permet tous les échanges. Réseaux sur lesquels, ce sont bien les nouveaux venus dans l'histoire des médias, les hébergeurs et les gestionnaires et détenteurs de l'accès à ces données qui vont imposer leur règne.

En définitive, nous sommes confrontés à un véritable paradoxe: jamais les données concernant l'information et l'actualité n'ont été aussi nombreuses et accessibles à un si grand nombre d'individus et jamais les modèles économiques des médias d'information et les pratiques des professionnels n'ont été autant questionnés et fragilisés.

\section{Les convergences}

Ce constat touchant l'entrée des médias mais aussi des pratiques culturelles dans l'univers du digital trouve un écho dans les analyses du chercheur nord-américain Henry Jenkins qui a souligné la dynamique de "convergence " que connaissent aussi bien les médias que les industries culturelles tout comme la plupart des pratiques sociales contemporaines, convergence qui a profondément remis en question le fonctionnement de ces dernières (Jenkins, 2013). Ce phénomène a donné le jour à un biotope digital au cœur duquel peuvent cohabiter toutes les données publiques ou privées relatives aux comportements et aux dire des individus et où la moindre activité humaine est tracée, localisée, archivée et calculée. On peut prolonger la réflexion du chercheur nord-américain en pointant certaines des retombées déterminantes de cet écosystème inédit et plus particulièrement celles qui touchent l'information en ligne. 
Un premier type de convergence purement technologique s'est rapidement imposé avec l'hégémonie du numérique et l'apparition de la toile et des réseaux sociaux qui autorisent désormais la circulation de tous les contenus possibles sur toutes les plateformes. Cette disponibilité globale des productions digitales a entraîné l'ubiquité et la disponibilité planétaire des médias et des sources d'information et a du même coup encouragé le nomadisme des lecteurs traditionnels. Elle a également entrainé des pratiques de braconnage crossmédia de la part de ces mêmes publics qui peuvent entrer, sortir et naviguer à leur guise de site en site. Simultanément, du côté des professionnels, ce même écosystème a initié une pratique transmédia de diffusion de l'information ; un journal en ligne proposant aussi bien de la radio, des interviews que des photographies ou de la vidéo, etc. Autre facteur accélérateur, celui de la miniaturisation, du faible coût et de la rapide banalisation des outils de production et de diffusion. Tous ces éléments ont considérablement accéléré et simplifié les mécanismes qui relient l'événement à sa diffusion et à sa réappropriation par des publics. Mais dans le même temps, ces mêmes facteurs ont durablement déstabilisé les modes de production et de circulation de l'information en reconfigurant les pratiques et les rôles des publics.

En effet, un second type de convergence concerne les pratiques des internautes et tient à la genèse d'Internet. La dimension de partage du réseau, conçu au départ comme un outil d'échange interpersonnel entre chercheurs, se perpétue dans la circulation des messages à travers une dimension informationnelle et encyclopédique mais aussi une dimension expressive et interactive. Dimension qui se coule sur une autre plus ancienne, le fait que ces technologies s'inscrivent dans une dynamique d'individualisation caractéristique de lévolution de nos sociétés. Pour paraphraser Louis Althusser, ces technologies “interpellent” l'individu, puisque l'interface médiatique se présente comme une plateforme individuelle et interactive tandis que le média demeure un média de masse. Cette « égalité des conditions » prophétisée par la pensée de la démocratie d'Alexis de Tocqueville semble réalisée dans l'accès de tous et de chacun à cet espace public ouvert et plastique que propose désormais le Web. Dans les sociétés développées, cette démocratisation de l'agir social du citoyen a été précédée par la dynamique sociétale qu’a lentement élaborée et accomplie la société de consommation à travers ses sommations et ses interpellations incessantes de l'individu-consommateur. A l'éga- 
lité politique tocquevilienne de toutes et de tous, cette dernière est parvenue à greffer et substituer un même marché pour tous, celui de la consommation de marchandises et de services par l'intermédiaire du choix individuel et personnel.

Aujourd'hui, il n'est plus question seulement du projet désincarné d'un individualisme égalitaire mais, comme le constate Danilo Martucelli, de la revendication d'une forme de singularisme à travers des séries d'expériences de reconnaissance et de mises à l'épreuve individuelles (Martucelli, 2010). Internet serait ainsi l'un des derniers rejetons et le territoire rêvé de l'accomplissement de cet « individu par excès » tel que l'a décrit Robert Castel produit du libéralisme et de l'économie de marché, territoire d'un nouveau type de socialité assignée à un individu désaffilié désormais des institutions (Castel, 2009).

Avec pour conséquence, l'intrusion et la coparticipation des internautes et donc de la société civile à la production de l'actualité, pratique virale qui fait planer une menace sur l'identité institutionnelle et professionnelle des acteurs de l'information. A titre d'illustration, aujourd'hui le médiascape féminin sur la toile se confond avec une nébuleuse de blogs, de sites, de portails, de chroniques sur You tube, etc., dispatchant et diffusant des rubriques et des contenus jusque là considérés comme le pré-carré des magazines féminins traditionnels. La presse féminine IRL (in the real life) a été une des premières victimes de cette concurrence expressive des profanes dans la production en ligne de news. Internet a réalisé un hold-up sur les nouvelles générations de lectrices potentielles des médias féminins. Cette « communauté imaginée » des femmes que les médias spécialisés avaient contribué à élaborer durant plusieurs décennies leur a été en partie dérobée. Avec pour conséquence principale le fait qu'une part de la légitimité des titres de la presse écrite féminine a migré, aux yeux des publics jeunes en particulier, au profit des bloggeuses de mode et de profanes aptes à faire part de leur expérience personnelle. Une partie du public de la presse féminine jusqu'alors captif s'est ainsi detournée de son géniteur. Pour limiter les dégâts, les leaders ont du cautionner l'entrisme de ces mêmes bloggeuses dans les magazines mais le plus souvent sans résultat patent.

Enfin, intervient un autre type de convergence d'ordre économique avec la stratégie d'intégration horizontale et la cannibalisation de différents médias par de nouveaux acteurs du soft power (les GAFA entre autres) qui démontre que l'économie de marché a au- 
jourd'hui définitivement anschlussé les industries culturelles et les médias. Le processus de désintermédiation de la pratique de nombreux pure players qui sétait esquissé a été confisqué par les gestionnaires de l'accès à la toile, les portails, les moteurs de recherche, les réseaux sociaux qui sont devenus les pourvoyeurs de visibilité dans l'univers digital et dans le même temps détenteurs de capitaux et de ressources décisifs (le rachat de You tube par Google). Ces nouveaux venus dans l'environnement des médias qui monopolisent les flux des internautes subtilisent une grande part de la valeur aux producteurs de contenus traditionnels. Ce biotope médiatico-culturel au cœur duquel cohabitent des distributeurs, des intermédiaires, des hébergeurs et des réseaux remet en question la propriété des produits tout comme les droits d'auteurs des créateurs de contenus.

Comme le constate Jeremy Rifkin « cette ère nouvelle voit les réseaux prendre la place des marchés et la notion d'accès se substituer à celle de propriété. Les entreprises et les consommateurs commencent à perdre contact avec la réalité fondamentale qui caractérisait la vie économique moderne -celle de léchange de biens sur un marché de vendeurs et d'acheteurs » (Rifkin, $2005: 10)$. Sur ce point, les législations globales sont toujours en chantier. Enfin, la circulation et la commercialisation de ces flux se déportent irrésistiblement vers le global avec une syndication des acteurs comme cest le cas pour le Huffington Post, Slate ou bien débouche sur leur concentration et leur absorption par de plus grands groupes IRL (le rachat de Rue 89 par le Nouvel Observateur).

\section{Continuités et discontinuités dans l'information en ligne}

L'univers digital incarne donc aujourd'hui un vaste écosystème qui est venu se greffer à l'univers de la communication publique et privée, avec une visibilité et un impact beaucoup plus considérables que dans d'autres secteurs qui ont subi pourtant les mêmes mutations : la banque par exemple (certaines banques ont fait disparaître l'argent physique), la santé (en France, la carte vitale a démonétisé en grande partie la tarification des soins), la bourse qui a fait disparaître tous les titres et aujourd'hui même les traders avec le trading haute fréquence géré entièrement par des robots, les agences de voyage remplacées par les algorithmes des comparateurs de prix, etc. Sans doute, parce que les bouleversements dans l'univers de la communication 
et de la culture concernent non seulement le mediascape quotidien du citoyen moderne mais aussi parce qu'ils sont peut-être annonciateurs, comme l'avance Dominique Cardon, « de formes inédites de partage du savoir, de mobilisation collective et de critique sociale " (Cardon, 2010: 8). Cet écosystème qui s'est introduit dans ces univers a pu assurer dans un premier temps une certaine continuité par rapport à l'ordre ancien, en s'accaparant un répertoire de pratiques et de services assurés par les médias et les productions académiques, il se singularise toutefois par des phénomènes de discontinuité.

La première continuité est celle de la plateforme et de ses coulisses que requiert chaque média qui pourrait avoir pour homologue dans l'histoire des médias traditionnels, l'industrie de l'édition et de la distribution. Contrairement à la vision contre-intuitive de la plupart des usagers, derrière la page écran, il existe toute une industrie de la toile. En partant des équipementiers qui développent des réseaux physiques ou des interfaces logicielles (Androïd, Microsoft, Apple...), des providers de connexion (Orange, Free...), des plateformes, moteurs, portails (Google, Facebook) ce n'est qu'au dernier étage de cette pyramide qu'on rencontre les producteurs de contenus et donc les médias en ligne (doublons de ceux du monde réel ou bien simples pure players). Cet écosystème n'est jamais à l'abri de turbulences de l'économie de marché et d'une véritable guerre de positions. L'oligopole que constitue la suprématie de quelques grands groupes rend stratégique (mais le plus souvent opaque) l'adossement de certains médias à ces derniers, comme ce fut le cas pour le Huffington Post racheté par AOL en 2011, lui même racheté en 2015 par Verizon. Derrière la course au trafic, cette part invisible de la navigation sur le web confisque l'essentiel de la valeur marchande aux détriments des véritables créateurs de contenus mais pèse aussi sur les orientations stratégiques des médias. Au cœur de ceux ci, cette puissance bouscule les enjeux civiques et déontologiques des journalistes. Il convient d'ajouter que le cadre fiscal mais aussi bien législatif que judiciaire tout à fait flou de cet empilement multinational ne facilite en rien les velléités d'intervention des pouvoirs politiques circonscrite au seul territoire des Etats-nations.

Il existe donc une infrastructure invisible et globalisée propre à cette nouvelle industrie, similaire à la production et à la distribution de marchandises qui ont constitué, au siècle dernier le socle de la société de consommation et le champ de bataille des entreprises multinationales. L'écosystème digital demeure donc indissociable d'autres 
systèmes, économique, capitalistique, législatif avec tous leurs effets secondaires. Et, ce sont désormais l'accès aux datas et la trajectoire des internautes qui constituent la matière première et la source de valeur de la net économie. Car celui qui parvient à se doter, le premier, du rôle d'agrégateur de données devient le leader d'un secteur de l'économie et va pouvoir prendre le dessus et déstabiliser durablement les détenteurs des places établies en annexant leur territoire ou bien en les réduisant à une portion congrue et cela à une vitesse vertigineuse. Google a terrassé ses concurrents en quelques années, ITunes d'Apple a fait de même avec les majors du disque alors qu'il était un intrus et un novice dans l'univers musical, Airnb et Booking.com fragilisent actuellement toute l'hôtellerie et Uber dérégule allègrement l'univers des taxis partout dans le monde. Pour la plupart de ces différentes plateformes la mise en relation des demandes des internautes à de simples bases de données et de services se sont transformées en prestation de services monnayables et en un véritable jackpot. Quant à l'univers de l'information sa désintermédiation et la délocalisation qu'il connait mettent à mal léconomie du secteur et fragilisent tout autant les producteurs et les éditeurs de nouvelles que la corporation même des journalistes.

Il existe une autre continuité repérable dans l'information en ligne, il s'agit bien sûr de la manne publicitaire qui permet —ou plutôt beaucoup le souhaiterait - le financement des sites. Certes, les quotidiens et les magazines de la presse écrite utilisaient déjà ce financement supplétif au coût réel du numéro. Mais, en ligne, c’est désormais le parcours effectif de chaque usager qui est pris en compte et qui devient monnayable auprès des annonceurs. Le plus étant sans doute aux yeux de ces derniers, une (auto) fragmentation des publics grâce à leur traçabilité sur les réseaux, leur géolocalisation et leur possible identification. A l'attention flottante et inerte du lecteur du magazine papier se substituent les traces de la trajectoire de l'internaute dans le site et éventuellement l'acquisition des contours de son profil de consommateur de news. D'une certaine manière un auto-ciblage accompli grâce à une mécanique indolore et invisible de cookies et d'algorithmes. C'est donc bien la course au nombre de pages vues et éventuellement le nombre de clics qui vont déterminer la valeur de l'investissement publicitaire dans chaque site. Et, tout bénéfice assuré pour les annonceurs, c'est le public qui va délivrer de lui-même son profil marketing, et à son insu le plus souvent, par l'intermédiaire de la traçabilité de son parcours même aléatoire. 
Comme certains le redoutent, la culture de l'accès tend inexorablement à « une marchandisation croissante de l'intégralité de l'expérience humaine » (Rifkin, 2005 : 129). Le rêve puéril mais manichéen de Walt Disney est désormais accompli ; les profils de tous les amis de mes amis sur Facebook sont mes amis et restent ad vitam oeternam des amis tout comme mes ennemis le demeurent à tout jamais. Aujourd'hui ce réseau social s'est transformé en un outil merveilleux d'irradiation publicitaire. Or, il tient à très peu de choses pour qu'il puisse incarner le cauchemar initié par la Stasi qui avait en sa possession tous les datas concernant mes amis mais à qui manquait la plateforme pour les gérer.

Cette dimension participative et intégrative et aussi cognitive de l'activité des internautes a d'autres types de retombées- le plus souvent l'insu de ces derniers. Du côté des médias, si la mise en ligne et la circulation d'informations et d'applications dédiées entraîne la traçabilité d'audiences, elle peut déboucher sur la constitution simultanée de communautés désormais capables de se connecter les unes avec les autres. Le public n'est plus un public mutique, ce " presque public » (Dayan, 2000) qu'avait initié et cartographié ("médiamétrisé") le petit écran, il est devenu un acteur du système et non plus une simple variable statistique. Cette reconfiguration des socialités publiques et privées liées à l'hégémonie croissante d'Internet nous pousse à une remise en cause simultanée et inéluctable tout autant de la notion de média que de celle de public.

\section{Une hétérogénéité énonciative et médiatique des sites de news}

Cette communication malléable et mobile impose donc de resignifier le rôle des acteurs sociaux dans la communication médiatique, tout autant celui des producteurs d'informations que celui des publics. Nous assistons à la fin du modèle du média homogène et monologal au profit d'une plateforme puzzle ou patchwork, polyphonique et hybride qui met en relations des données et des trajectoires d'internautes. Sur le plan des contenus, les trois étapes classiques de la production médiatique - celle de la création/production, de la distribution/circulation et de la réception/appropriation - telles que Stuart Hall les définissait ne sont plus étanches (Hall, 1997). Traditionnellement, cantonnés à l'étape de réception, les non journalistes participent de plus en plus à la circulation, voire à l'étape de la production de 
l'information. Comme le constate Henri Jenkins un média n'est plus simplement une courroie de transmission entre producteurs, textes et publics mais " un flux reliant les uns aux autres des acteurs sociaux » (Jenkins, 2013 : 16). Simultanément, le rubricage traditionnel et le modèle éditorial pyramidal caractéristiques de la presse semblent durablement remis en cause au profit d'un jeu soutenu d'intertextualité, de polyphonie et de phénomènes d'intermédialité.

Concernant la hiérarchie de l'information dans le site, c'est en définitive le lecteur qui l'établit et c'est lui qui va générer sa propre actualité et le plus souvent à l'intérieur du flux de plusieurs médias. Le journal en ligne s'est donc transformé en un texte-parcours qui repose sur cette textualité réticulaire qui s'autogénère au fur et à mesure de la navigation de l'internaute. Lécrit académique, le flash info de la radio tout comme l'édition du journal télévisé encadraient la réception de manière unilatérale, non négociée et linéaire et faisaient entrer les usagers dans un véritable tunnel dont ils ne pouvaient modifier ni les normes ni le cadre préétabli. Un nouveau type de textualité apparaît avec Internet, une textualité navigante, à partir d'une interface mosaïque et tabulaire permettant l'accès à des modules indépendants qui obéissent le plus souvent à des temporalités distinctes.

En conséquence, la plupart des sites d'information vont chercher à anticiper ces modalités inédites de la réception en ligne. C'est le cas pour le Huffington Post pour qui une fraction de la homepage se reconstitue en permanence en fonction de cette hiérarchie que la navigation des lecteurs impose en temps réel. Il ne s'agit plus de la page vitrine comme l'incarnait la Une statique du quotidien papier, gravée dans le marbre, la veille de la parution. Les trajectoires des internautes tout comme les mécanismes d'indexation qui les accompagnent aujourd'hui aboutissent à une sorte googelisation des news qui régit la mise en ligne des pages-écrans. Si cette scénographie délinéarisée qui sécrit désormais dans l'espace et le temps doit constamment se plier à la textualité navigante en temps réel des internautes, elle se doit également de demeurer toujours ouverte aux aléas de l'actualité événementielle. C'est un des points forts des médias en ligne qui sont capables d'assurer la gestion de temporalités hétérogènes qui peuvent irriguer le flux des nouvelles, du document d'archive du fast checking jusqu'au présent live du « surgissement de lévénement » (Soulages, 2001).

Cette remise en question indirecte du champ monolithique des professionnels s'accompagne d'une exigence de réflexivité et de trans- 
parence sur la production de l'information, explicite souvent dans le processus de médiatisation lui-même et surtout dans un élargissement des opérations énonciatives aux membres de la société civile qui peuvent devenir des acteurs critiques et aussi la matière et la source même de l'information. La charte du Huffington Post milite du reste pour cette association volontariste des contenus médiatiques "légitimes" et des paroles profanes donnant le jour à un format hybride constitué d'articles de ses rédacteurs et de blogs de personnalités de la société civile. De plus, les internautes ont la possibilité de commenter, d'interroger et de vérifier l'information d'où le pouvoir grandissant et la pression du fast checking et cette capacité de participer et de réagir qui caractérise désormais l'usager des médias numériques.

\section{Vers une nouvelle définition des publics}

Car si ces pratiques reposant sur l'accès et la mise en réseau des internautes ont permis l'apparition de nouvelles ressources cognitives partagées comme celles à l’origine de Wikipédia misant sur le travail et l'intelligence collaborative, cette incursion de l'internaute dans l'espace public peut parfois se métamorphoser en une posture contre-hégémonique et en une critique indirecte du fonctionnement média-référencé du champ journalistique. Elle a donné le jour à ces " communaux collaboratifs » évoqués par Jérémy Rifkin (2014) caractérisés par l'intervention de collectifs ou d'individus apportant le flux de leurs propres références voire de leurs expériences ou expertises (Wikileaks). Elles constituent des sources d'information alternatives qui s'auto-légitiment dans le partage des mêmes valeurs et d'une même volonté d'indépendance sur lesquelles repose la liberté d'information dans les sociétés démocratiques. Cet entrisme des publics dans l'univers des médias de masse et plus particulièrement des médias d'information bouscule le fonctionnement de ces derniers qui se sont développés dans un contexte de stricte séparation entre l'expression des socialités personnelles et l'espace public.

Endossant le rôle de gate-keeper, éditeurs ou journalistes, ont longtemps contrôlé la frontière entre les deux mondes. Grâce à leur accès au réseau des réseaux, s'offre aujourd'hui aux membres de la société civile une possibilité d'intervention inédite dans la sphère publique en contournant ces médiateurs organiques. De consommateur de l'information, l'usager est en mesure de devenir un agent 
médiatique, à part entière. "Don't hate the media, become the media" comme l'a proclamé Jello Biafra. Cela s'accompagne de l'apparition de nouvelles modalités socio-techniques d'expression désintermédiées, blogs, posts, tweets, etc., à l'initiative de profanes. Ces pratiques créatives et participatives de commentaire voire de création de contenus sont orientées vers la recherche d'information mais aussi sur le partage de celle-ci entre pairs.

Cette cohabitation d'articles de la rédaction et de blogs à l'initiative d'acteurs extérieurs tout comme le spectre thématique très large des contributions - de l'article people au blog-chronique d'Obamasont une des singularités revendiquées par certains pure players comme les créateurs du Huffington Post. Lapparition de ce type de média hybride valide l'énoncé provocateur émis par John Fiske qui parlait en son temps de "démocratie sémiotique » (Fiske, 1987) pour qualifier la néo-télévision en pointant les stratégies empathiques et réflexives de certaines productions dans lesquelles le public devenait décisionnaire, ce qui semble être aujourd'hui réalisé quotidiennement par les millions de followers sur la toile.

Du reste, un parallèle peut être établi entre la création du Huffington Post et l'apparition au début des années 1990 en France des émissions omnibus ou les talk-shows " patchwork » de la télévision qui cherchaient à agréger des publics hétérogènes (Nulle part ailleurs, ou le Grand Journal), comme ce fut le cas lors de la création de Canal+, un pure player dans le PAF des années 80 (Soulages, 1994). En optant pour une stratégie de contre programmation à travers cette production omnibus, il s'agissait alors de capter un public composite, plus jeune et nomade, susceptible d'entrer et de quitter le spectacle à tout moment. Cet embedment des publics est aujourd'hui en quelque sorte réalisé en partie sur la toile où l'internaute peut participer au storytelling de l'événement avec l'intégralité et le remake infini de son déroulement et aussi de son effacement. Le risque étant que journalistes et public prennent chacun leur part à la même fiction et s'enferme dans celle d'une unique version de la vérité, celle du buzz et du plus grand nombre. En définitive, le web a placé le citoyen au centre des flux de l'information mais pour autant ce dernier est-il au cœur des événements?

Ces mutations du statut et des rôles échus au public, nous pousse à interroger cette notion ambivalente de public qui a accompagné l'essor des médias de masse. Comme David Morley et d'autres cher- 
cheurs l'avaient déjà pressenti elle n'est peut-être et depuis très longtemps qu'une simple fiction politique ou statistique (Morley, Dayan, 1993). Fiction idéologique durable qui était celle induite par le poids d'un Etat-nation centralisateur et autoritaire qui faisait face à un peuple homogène et mutique. Cette notion discutée de public, notion politique au départ, née au cœur de l'agora grecque, est devenue une variable statistique, celle d'audience avec le règne des médias de masse. Comme le relève Henry Jenkins, c'est sans doute la relative stabilité de la presse imprimée ou de la télévision, qui n’a duré que quelques siècles pour la première et quelques décennies pour la seconde, qui nous a incité à substantialiser et à éterniser le fantasme d'un public stable et toujours homogène.

Mais à cette conception purement statistique, s'en greffe une nouvelle. Cette dimension pragmatique et agrégative du média avait déjà été évoquée il y a plus d’un siècle par Gabriel Tarde qui soulignait le poids de la conversation et du bouche à oreille dans la production de l'opinion. Aujourd'hui, cette pragmatique sociale se nourrit des pratiques collaboratives de la net économie qui seraient aussi une manière de faire société et de construire du lien social, celui de " communautés imaginées ", à mi-chemin entre le public au sens habermassien et les publics diasporiques d'Arjun Appaduraï. Avec pour conséquence en retour, l'individualisation et la dynamique communautaire de plus en plus poussée des usagers de ces nouveaux médias en phase avec les concepts de «mediascape» (Appaduraï, 2005) et de "communauté imaginée » de Benedict Anderson (2002). La société civile s'immiscerait ainsi sur la scène de l'espace public en bousculant les chasses gardées des corporations et des professionnels légitimes, étape annonciatrice, aux yeux de certains, de nouvelles formes de socialisation mais aussi de nouvelles formes politiques (Cardon, 2010).

\section{Le temps des usages}

A l'aube de la révolution des réseaux, Patrice Flichy discernait dans les innovations techniques qui avaient marqué le champ de la communication depuis près de deux siècles, cinq temporalités — « le temps technique, le temps industriel, le temps de lévolution des métiers, le temps esthétique et le temps des usages »(Flichy, 1987). Il constatait que « le décalage entre l'invention technique et son usage 
industriel vient du fait qu'il faut incorporer dans la technique des savoir faire sociaux qui la rendent appropriable par une société particulière. Une technique n'est utilisable que dans un mode d'organisation sociale donnée. " L'usage du smartphone s'est ainsi ritualisé, petit à petit, par sa sortie de l'usage corporatiste des seuls cadres (le BlackBerry) et élargi grâce à une synergie entre un usage démocratisé et ouvert à de multiples applications et une technologie nomade. Petit à petit, le temps des usages et le temps esthétique ne font plus qu'un. L'adoption de nouveaux usages par les publics correspond à l'apprivoisement réussi d'une technologie et garantit l'existence d'un marché commercial.

Simultanément, dans l'univers du numérique, les pratiques des agrégateurs qui, au départ s'apparentaient à une forme de braconnage à l'initiative d'innovateurs solitaires se sont petit à petit institutionnalisées pour donner naissance à des oligopoles monétisant les usages et le trafic des audiences. Linnovation technique se transforme rapidement en industrie et en flux financier, avec pour conséquence une obligation pour la profession : celui de l'articulation entre métiers anciens et métiers nouveaux. Les médias et les journalistes se trouveraient aujourd'hui dans cet entre deux, écartelés entre plusieurs temporalités, celle des usages et celle de l'industrie et face à la pression de cette dernière, celle de lévolution inéluctable de leurs pratiques. Comme toujours, le « temps des usages » est parvenu une fois de plus à court-circuiter « le temps de lévolution des métiers ». Le journaliste web, s'il ne veut pas se cantonner à une pratique proche du journalisme de collecte ou de reprise doit de toute évidence se repenser.

Quant à la notion de public, sa nature et son rôle, se retrouvent bousculés par les nouveaux modèles de la communication médiatique établis sur un mode de diffusion original, mass médiatique mais avec une architecture de communication de masse qui repose sur une interface personnelle. On peut alors ne trouver rien détonnant à ce que les individus s'emparent d'une technologie laissée à leur disposition à portée de main (dans leur poche) dans les coulisses de l'espace privé et ouvert à des protocoles de désintermédiation et de nomadisme a contrario, le théâtre ou le cinéma sont structurellement des dispositifs bâtis sur la médiation et l'existence d'un public et d'un dispositif sédentaire et délocalisé. Quant à la posture du chercheur, de celui qui refuse d'enfiler l'uniforme du chercheur organique boursouflé par sa monodiscipline chroniquant le flux médiatique, il est urgent qu'il 
abandonne le prophétisme ou le catastrophisme technologique pour chercher à dépasser les oppositions classiques entre technologisme et sociologisme et proposer une approche " sociotechnique » considérant que les usages des dispositifs numériques sont façonnés à la fois par des normes sociales et des configurations techniques.

\section{Références bibliographiques}

ANDERSON Benedict, L'imaginaire national. Réflexions sur l'origine et l'essor du nationalisme, Paris, La Découverte/poche, 2002.

APPADURAI Arjun, Après le colonialisme. Les conséquences culturelles de la globalisation. Paris, petite bibliothèque Payot, 2005.

CARDON Dominique, La démocratie Internet. Promesses et limites, Paris, Seuil, 2010, p. 8.

CASTEL Robert, La montée des incertitudes. Travail, protections, statut de l'individu, Paris, Seuil, 2009.

DAYAN Daniel, «Le presque public », Réseaux n 100 , Paris, CNET, 2000, p. 427-456.

FISKE John, Television Culture, Londres, Routledge, 1987.

FLICHY Patrice, Le sacre de l'amateur. Sociologie des passions ordinaires à l’ère numérique, Paris, Seuil, 2010.

FLICHY Patrice. «Communication : progrès technique et développement des usages ». In: Réseaux, 1987, volume 5 n² 24. pp. 99-100.

HABERMAS Jürgen. « L'espace public, 30 ans après. » In: Quaderni. N. 18, Automne 1992. Les espaces publics. pp. 161-191.

JENKINS Henry, La culture de la convergence. Des médias au transmédia. Paris, Armand Colin, Ina éditions, 2013. 
MARTUCCELLI Danilo, La société singulariste, Paris, Armand Colin, 2010.

MEAD Margaret, Le fossé des générations, Paris, Denoël, 1971.

MORLEY David \& DAYAN Daniel, " La réception des travaux sur la réception » Retour sur «Le Public de Nationwide », Hermès 1993/1 n ${ }^{\circ}$ 11-12, p. 31-46.

RIFKIN Jeremy, Lâge de l'accès. La nouvelle culture du capitalisme. Paris, La Découverte poche, 2005.

RIFKIN Jérémy, La nouvelle société du coût marginal zéro. L'internet des objets, l'émergence des communaux collaboratifs et l'éclipse du capitalisme, Paris, Les liens qui libèrent, 2014.

SIMONE Raffaele, Pris dans la Toile. L'esprit au temps du web. Paris, le débat Gallimard, 2012.

SOULAGES Jean-Claude \& LOCHARD Guy, " Les scénarisations visuelles ", in Charaudeau Patrick (dir.) La télévision et la guerre. Déformation ou construction de la réalité? Le conflit en Bosnie (19901994), Bruxelles, De Boeck / INA, 2001.

SOULAGES Jean-Claude, LOCHARD Guy “ Les imaginaires de la parole télévisuelle ; permanence, glissements et conflits", Réseaux $n^{\circ} 63$, Paris, CNET, 1994, pp. 15-38.

STUART Hall " Codage/décodage ", Sociologie de la communication, Paris, CNET, 1997, p. 59-72, [« Encoding / decoding model of communication », 1973]. 